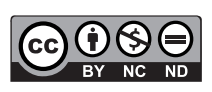

Revista EIA

ISSN 1794-1237

e-ISSN 2463-0950

Año XVIII/ Volumen 18/ Edición N.35

Enero-Junio de 2021

Reia35003 pp. 1-20.

Publicación científica semestral

Universidad EIA, Envigado, Colombia

\section{Para citar este artículo /} To REFERENCE THIS ARTICLE /

García-Capdevilla, D.A.; López-De

Parra, L.; Millán-Rojas, E.E. (2021).

Contexto y condiciones del eco

marketing para promocionar el

turismo de naturaleza. Revista EIA,

18(35), Reia35003 pp. 1-20.

https://doi.org/10.24050/reia.

v18i35.1419

\section{Autor de correspondencia:}

García-Capdevilla, D.A. (Diana Alí): Doctorado en Educación y Cultura Ambiental de la Universidad de la Amazonia, Colombia.

Correo electrónico:

dia.garcia@udla.edu.co

Recibido: 05-05-2020

Aceptado: $28-10-2020$

Disponible online: 01-01-2021

\title{
Contexto y condiciones del eco marketing para promocionar el turismo de naturaleza
}

\section{Diana Alí García-CAPDEVILla ${ }^{1}$ LILLYAM LÓPEZ-DE PARRA ${ }^{1}$ EDWIN EDUARDO MILLÁN-ROJAS ${ }^{1}$}

1. Doctorado en Educación y Cultura Ambiental de la Universidad de la Amazonia, Colombia

\section{Resumen}

El objetivo del trabajo fue determinar el contexto y las condiciones del eco marketing para potencializar el turismo de naturaleza. La metodología fue descriptiva, mixta y método estudio de caso. La población fue de 41 empresarios y dos directivos. Se aplicaron encuesta, entrevistas a profundidad y grupo focal. Se analizaron datos con el software SPSS y triangulación. Los resultados obtenidos demostraron que el eco marketing, la economía ecológica, la formación y la ejecución de prácticas amigables son requeridos para ofertar este tipo de servicios turísticos. Por esa razón, se concluye que el eco marketing minimiza efectos negativos en el ambiente y potencializa el turismo de naturaleza.

Palabras Claves: Estrategias de marketing, Turismo de naturaleza, Conservación del ambiente, Sustentable.

\section{Context and conditions of eco marketing to promote nature tourism}

\section{Abstract}

The objective of the work was to determine the context and conditions of eco-marketing to enhance nature tourism. The methodology was descriptive, mixed and case study method. The population was 41 entrepreneurs and two managers. A survey, in-depth interviews and a focus group were applied. Data was analyzed with SPSS software and triangulation. The results obtained showed that ecomarketing, ecological economics, training and the implementation of friendly practices are required to offer this type of tourism services. For this reason, it is concluded that ecomarketing minimizes negative effects on the environment and enhances nature tourism.

Key Words: Marketing strategies, Nature tourism, Environmental conservation, Sustainable. 


\section{Introducción}

El propósito de este trabajo es presentar un aporte para potencializar el turismo de naturaleza de manera sustentable a través del eco-marketing. En este sentido, el objetivo de este artículo de investigación fue determinar el contexto y condiciones del eco marketing del turismo de naturaleza. El turismo convencional, aunque es más competitivo, dinámico y genera ingresos para el desarrollo económico de la sociedad puede desencadenar la depredación de los destinos turísticos, en las temporadas altas por la gran demanda de personas, transporte e infraestructura. Esto conlleva al desgaste de los lugares y al incremento de la inflación, la cual se genera por el ingreso de turistas con alto poder adquisitivo y el encarecimiento de los productos y servicios de la zona. Además, contribuye al deterioro ambiental y a la deforestación cuando se implementa en zonas ricas en bosques; es decir, impacta de forma negativa la biodiversidad de la región (Gómez, 2017).

Ante esta situación, en la actualidad, se impulsa el turismo de naturaleza en la perspectiva de lograr en primer lugar, el conocimiento y disfrute del ambiente rural, la conservación responsable de los ecosistemas y, en segundo lugar, la obtención de ingresos económicos en pro de una mejor calidad de vida de los habitantes (Gil, 2019). No obstante, estas ventajas en algunos territorios no se cumplen dichas expectativas.

Una de las razones, pese a la belleza de los escenarios naturales es su poca divulgación y, por ende, la difusión de estos atractivos turísticos no se efectúa a través de la implementación de las Tecnologías de la Información y la Comunicación (TIC). Asimismo, los promotores de la actividad no poseen conocimientos acerca de las ventajas de su fomento mediante el eco marketing, que puede traer los turistas llamados cazadores de experiencias únicas (García, y Salvaj, 2017).

$\mathrm{Al}$ respecto, en el ámbito internacional varios investigadores se han interesado por la problemática que afronta el desarrollo del eco marketing. Monteiro, et al. (2015), en un estudio cualitativo de carácter exploratorio abordaron la fundamentación teórica de los conceptos de marketing, sus acciones, la presentación y las características de la combinación de marketing adaptada al marketing verde. Concluyeron que esto plantea desafíos para aplicar estrategias de acuerdo al entorno y alcanzar mercados amplios a nivel nacional e internacional.

Asimismo, en la investigación de López, y Moya (2015), se analizó el marketing ecológico como una estrategia para mejorar la percepción sobre la minería del carbón en la región zuliana, desde la perspectiva teórica de varios autores estudiosos de la temática (Chamorro, 2001) (Calomarde, 2000) (Lorenzo, 2002) (Machín, 2007) (Vicente, y Ruiz, 2002). Se empleó la metodología descriptiva; la muestra definida (unidades informantes) estuvo conformada por 34 supervisores del área de producción de las empresas. Las conclusiones indicaron que el marketing ecológico del carbón en este sector se realiza de manera óptima, mediante la identificación de los elementos resultantes de la combinación del marketing y el eco marketing, factores y estrategias determinantes para mejorar los procesos en esta clase de organizaciones.

En México, se verificó la influencia del eco marketing en las tendencias de consumo y su posibilidad para posicionar estratégicamente los productos en el mercado (Castañeda, 2017) (Bueno-Solano, Wendlandt-Amézaga, y ValenzuelaReynaga, 2018).BCon la visión de complementariedad, Córdova (2012) planteó el análisis y evaluación de la perspectiva estratégica empresarial, con el objetivo de realizar la gestión conjunta del marketing ecológico y el turismo sostenible en la provincia del Tungurahua. 
En Colombia, Díaz, y Moya (2016), en un tipo de estudio descriptivo, con la metodología cuantitativa, determinaron el impacto de las actividades de green marketing aplicadas por las empresas del sector de la tecnología. Específicamente, en los procesos desarrollados para la creación de valor de marca y en la percepción de los consumidores. Establecieron la importancia del green marketing en la consecución del modelo de Brand Equity, fundamentado en la comunicación efectiva y, el intercambio de opiniones con los consumidores para contribuir a la búsqueda de problemáticas ambientales.

No obstante, en estos trabajos, es clara la ausencia de investigaciones con enfoque hacia la implementación de las estrategias de eco marketing para lograr el posicionamiento del turismo de naturaleza, el desarrollo ambiental sustentable y, la generación de oportunidades laborales. Asimismo, para tener en cuenta las características de los grupos generacionales, los cuales se convierten en actores importantes y a la vez, clientes potenciales (Díaz-Sarmiento, López-Lambraño, y Roncallo-Lafont, 2017).

Por otra parte, en el contexto de la región de la Amazonia colombiana, eje de conservación de la biodiversidad mundial, uno de los grandes problemas ambientales es el alto índice de deforestación, con el 75\% del total nacional (Instituto de Hidrología, Meteorología y Estudios Ambientales - IDEAM, 2019). En esta región, sobresale, de manera particular, el Caquetá; departamento que ocupa el primer lugar en la deforestación de los bosques (IDEAM, 2019), lo que puede generar desastres naturales y humanos (López-Vargas, y Cárdenas-Aguirre, 2018). Aunque, el turismo de naturaleza puede ser una de las posibilidades para fomentar su valoración, se desconoce el pensamiento de los empresarios del Caquetá sobre el eco marketing como una estrategia de promoción del turismo de naturaleza y como alternativa amigable con el ambiente. En este sentido, el interrogante a resolver es: ¿cuál es el contexto y cuáles son las condiciones del eco marketing para promocionar el turismo de naturaleza en el Departamento del Caquetá?

Resolver el anterior cuestionamiento se justifica porque se busca el reconocimiento del turismo como parte de la mundialización territorial sustentable con la intervención e interrelación de factores sociales, tecnológicos, económicos y políticos. Se apuesta a la producción de cambios estratégicos en la sociedad; en particular, en Colombia a partir de la firma del Acuerdo de Paz y en la fase posterior de pos-acuerdo; en esta perspectiva, se busca el fomento del turismo de naturaleza, el cual es considerado un producto sombrilla con posibilidades de potencializarse mediante el eco marketing (Presidencia de la República de Colombia, 2016).

$\mathrm{Al}$ respecto, se considera primordial indicar que el marketing es importante para cualquier organización; más aún, si se utiliza de manera adecuada la investigación de mercados como herramienta para la toma de decisiones. Es así que, el mercadeo se define como el desempeño de actividades de negocio que dirigen el flujo de bienes y servicios desde el fabricante hacia el consumidor (Asociación Americana de Marketing - AMA, 1960).

La AMA ${ }^{1}$ abordó el marketing desde una perspectiva técnica; es decir, se centra en las herramientas del marketing mix, definido como "la mezcla de cuatro elementos que van a suponer la efectiva ejecución de una estrategia de marketing" (Fernández, 2015, p. 66). De modo similar, Kotler, y Armstrong (2012) expresan que estos elementos son las 4P's, comprendidas como: a) producto, b) precio, c) promoción y d) plaza.

1 AMA: se empleará en adelante, para referirse a la Asociación Americana de Marketing 
Luego, se pasó a entender la actividad de marketing como un proceso susceptible de ser desarrollado también, por organizaciones no lucrativas (Talavera, 2017). Así, se estableció que el marketing es el proceso de planificación y ejecución del concepto de promoción, precio y distribución de ideas, bienes y servicios para crear intercambios que satisfagan objetivos individuales y colectivos (Sánchez, y Montoya, 2016).

Históricamente, el eco marketing, surge en la década de los 90. En las empresas es definido como un proceso que ejecuta acciones para obtener productos o servicios de manera amigable, al reducir los daños que se puedan ocasionar al ambiente (Castañeda, 2017) (Castellano, y Urdaneta, 2015). Los autores (Dima, \& Vlăduțescu, 2012) (Vlăduțescu, 2012) (Macris, 2013) explican que, el movimiento ecológico apareció y se desarrolló como una reacción al impacto negativo que las actividades humanas tienen sobre el ambiente y, como consecuencia de las exigencias de los consumidores de crear productos que representaran el menor impacto negativo en el ambiente. Se busca evitar así, los desastres naturales producto de la destrucción acelerada del mismo. En España, el eco-marketing fue objeto de estudio científico mediante la evaluación de la importancia de este término como línea de investigación (Chamorro, Miranda, y Rubio, 2006).

También es denominado marketing ecológico, marketing verde, marketing ambiental o green marketing, se cimienta en el desarrollo de una conciencia positiva hacia la conservación de la naturaleza, con cambios en los procesos productivos y la promoción innovadora de bienes y servicios. Fuller, \& Butler (2015) destacan el marketing ecológico como un proceso de gestión estratégica integral y responsable en el que se identifican, anticipan y satisfacen las necesidades de las partes interesadas para obtener una recompensa razonable; sin afectar negativamente, el bienestar ambiental, humano o natural. Por tanto, si las necesidades y aspiraciones de la sociedad neoliberal no se satisfacen plenamente, se genera el aumento en el nivel del consumismo insostenible por parte de los integrantes de la comunidad, quienes lo idealizan con la mejora en la calidad de vida. Sin embargo, desconocen los efectos negativos para la naturaleza; de ahí, la importancia adquirida por el eco marketing en la sociedad.

En el marco de la dinámica del mercadeo se han identificado necesidades humanas y sociales para crear tendencias hacia marcas ecológicas y lograr un destacado posicionamiento (Kotler, y Keller, 2006) (Baker, \& Sinkula, 2005) (De Ruyter, De Jong, \& Wetzels, 2009) (Grinstein, y Nisan, 2009). Esto se sustenta porque cada día son más los consumidores que otorgan mayor importancia a las características medioambientales de los productos y servicios demandados. El propósito es desencadenar nuevas oportunidades de negocio (Durmaz, \& Sevil, 2011); éstas deben originarse desde la responsabilidad social empresarial, para causas sociales y medioambientales (Rubio-Guerrero, 2016).

Por otra parte, la metodología que se empleó para la realización de este trabajo fue descriptiva y mixta. El método fue el estudio de caso. La población estuvo constituida por 88 empresas de la región dedicadas al fortalecimiento y promoción del turismo y actividades ecoturísticas. De la base de datos a diciembre de 2018, obtenida en la Cámara de Comercio de Florencia, se extrajo una muestra representativa de 21 empresas. La muestra se definió mediante la técnica del muestreo aleatorio simple. La recolección de los datos cuantitativos se efectuó mediante la técnica de la encuesta realizada a los 21 empresarios; el instrumento fue el cuestionario, elaborado con preguntas de carácter dicotómico. Los datos de carácter cualitativo fueron recogidos a través de las técnicas del grupo focal con la participación de 20 empresarios y las entrevistas a profundidad a dos directivos de entidades importantes del departamento de Caquetá. 
En el artículo se presentan resultados relacionados con el contexto y las condiciones requeridas para la promoción del eco marketing, a fin de potencializar el turismo de naturaleza en la región de la Amazonia colombiana; específicamente, en el departamento del Caquetá. Se estructuró en los siguientes apartados: introducción, metodología, resultados y discusión, se formularon los resultados del eco marketing relacionados con: el contexto, conceptos de los participantes sobre eco marketing, estrategias para las empresas, inversión de recursos, oportunidades para invertir, valoración del eco marketing y del turismo de naturaleza, potencialidades y progresos del turismo de naturaleza. Además, se analizó la relación con la Economía EcológicaSF. Finalmente, se expusieron las conclusiones.

\section{Materiales y Métodos}

Esta investigación es de tipo descriptivo, método estudio de caso; se elaboró mediante la adopción del enfoque mixto con la aplicación de técnicas para la recolección de datos de carácter cuantitativo y cualitativo. Acorde con el objetivo el propósito fue el de obtener la información necesaria acerca de la percepción del eco marketing de los empresarios de la región y el grado de interés para implementar estrategias de marketing que puedan fomentar el turismo de naturaleza (Malhotra, 2016) (Hueso, y Cascant, 2012) (Bradley, 2013) y, determinar de esta manera, el contexto y condiciones del eco marketing del turismo de naturaleza.

La exploración de datos primarios, originados en la investigación con el fin de abordar el problema, se recopilaron con base en: las percepciones, conocimientos y preferencias de las unidades de información. Los secundarios, fueron obtenidos en libros de autores reconocidos, artículos de revistas científicas, documentos institucionales, investigaciones, informes de entidades nacionales e internacionales. La búsqueda exhaustiva de información se efectuó en bases de datos reconocidas como: Web of Science, Science Direct, Scopus, Proquest, entre otras. Dicha información se clasificó de acuerdo con la relevancia, precisión, actualidad, imparcialidad y según las necesidades del proyecto (Kotler, y Armstrong, 2017) (Serrano-Abreo, y Castellanos-Granados, 2019).

Para definir la muestra poblacional se utilizó la fórmula de muestreo aleatorio simple. Mediante esta, se extrajo al azar la muestra de la población, se eligió cada uno de los individuos mediante números aleatorios, acorde con el empleo del método de implementación de la tabla de números aleatorios en el sistema (Hair, Bush, y Ortinau, 2010) (Hernández, Fernández, y Baptista, 2014). Este tipo de muestreo garantizó que cada unidad en la población objetivo tuviera la misma oportunidad de ser seleccionada. De acuerdo con Otzen, y Manterola (2017) y Ñaupas, Mejía, Novoa, y Villagómez (2014) es un método simple y eficaz, en donde todos los individuos hacen parte del universo. Para esta investigación, la muestra se obtuvo de la base de datos de empresas constituidas en el departamento del Caquetá (Colombia) hasta diciembre de 2018 y, registradas en la Cámara de Comercio de Florencia, su capital. En esta base, se da cuenta de 88 empresas relacionadas con la realización de acciones ecoturísticas, en pro del impulso y desarrollo del turismo. La muestra representativa fue de 21 empresas; a sus empresarios, se les aplicó la encuesta a través del correo electrónico, una vez aceptaron dar su consentimiento.

El instrumento de la encuesta fue un cuestionario estructurado en un formulario en Drive, con 17 preguntas dicotómicas de selección múltiple; es decir, aquellas en las que se puede seleccionar una de las dos opciones: sí o no (Niño, 2011) (Peña, Serra, y Cardona, 2017). Esta técnica es útil porque permite al encuestador indagar 
con mayor libertad y trabajar con objetividad. En general, se diseñó para interrogar a los participantes, solicitar opiniones específicas y obtener datos confiables (Malhotra, 2016). A este proceso se le hizo seguimiento oportuno, con el fin de lograr el diligenciamiento en el término requerido para el desarrollo de la investigación. Al finalizar la etapa de recolección de la información se realizó la revisión de las respuestas consolidadas en la respectiva hoja de cálculo de Excel; estas fueron la base para el respectivo análisis detallado de los resultados obtenidos.

Por otra parte, las técnicas cualitativas de recolección de información implementadas fueron el grupo focal y las entrevistas a profundidad (se estructuraron en cuatro momentos: entrada, inicio, desarrollo y cierre). Estas técnicas fueron aceptadas por los participantes mediante la firma del consentimiento informado. De igual forma, los cuestionarios como instrumentos de cada una de ellas, previamente, fueron objeto de pilotaje y validación de expertos.

Las entrevistas se aplicaron a dos directivos: el Presidente Ejecutivo de la Cámara de Comercio de Florencia y al Coordinador de Cultura y Turismo Departamental. Para tal efecto, se diseñó un guion acorde con el campo de acción de los individuos y sus habilidades en los respectivos cargos. El propósito fue la profundización del tema planteado mediante preguntas estratégicas (Trespalacios, et al., 2016). A través de esta técnica los entrevistados suministraron información relevante con respecto a sus experiencias y conocimientos referentes a la promoción del turismo; en particular, en el de naturaleza.

Así mismo, el grupo focal se organizó con el objetivo de determinar aspectos importantes para diversos empresarios del departamento de Caquetá y establecer el grado de conocimiento acerca del eco marketing como estrategia novedosa en la potencialización del turismo de naturaleza en la región. La finalidad fue garantizar la recolección de información valiosa a través de esta técnica, adoptada por el marketing en los años 70, mediante la formulación de debates sobre un tema deseado (Cruz, et al., 2017).

$\mathrm{Al} \mathrm{respecto,} \mathrm{el} \mathrm{grupo} \mathrm{focal} \mathrm{es} \mathrm{la} \mathrm{técnica} \mathrm{cualitativa} \mathrm{más} \mathrm{empleada} \mathrm{en} \mathrm{la}$ investigación de mercados y tiene gran aceptación por la dinámica creada por el moderador en el momento de su ejecución (Malhotra, 2016). Las fases de su implementación fueron: a) planificación del grupo, b) incorporación de los participantes, c) moderación de las reuniones y, d) informe de conclusiones. De acuerdo con lo anterior, se efectuó la selección de los empresarios del sector turístico para la conformación de dos grupos, cada uno de diez empresarios del departamento de Caquetá. Se contactaron de manera presencial para concitar su participación mediante la explicación del objetivo de las reuniones. Durante las sesiones, se designaron moderadores encargados de diseñar las estrategias para acercarse a los invitados, generar confianza y obtener resultados útiles en el análisis de la investigación. Asimismo, se plantearon preguntas acerca de: a) sus viajes, b) potencialidades del turismo de naturaleza en el Caquetá, c) nivel de conocimiento sobre eco marketing, d) la percepción sobre diferentes aspectos turísticos y e) las experiencias vividas en sus travesías.

El análisis de los datos se efectuó mediante la técnica de triangulación de datos; esta hace referencia al uso de diversas fuentes de información y estrategias sobre eco marketing y turismo de naturaleza (Aguilar, y Barroso, 2015). Igualmente, se utilizó la triangulación metodológica, basada en la combinación de métodos de recolección y análisis de datos para comprender la realidad del tema investigado (Instituto de Estudios Sociales de Andalucía, 2012). En este sentido, la aplicación de estas técnicas, se conoce como triangulación múltiple. Por otra parte, como técnica para la sistematización y análisis de datos, se utilizó el software estadístico Startical 
Product and Service Solutions-SPSS para el procesamiento y revisión de la información recolectada con los instrumentos en los diferentes contextos, elaboración de la matriz de datos, correlación de variables, construcción de tablas y figuras. La finalidad fue la generación en la confiabilidad de los datos y, la obtención de consistencia, complejidad y profundidad en el estudio y en sus hallazgos (Patton, 2002). Las técnicas empleadas facilitaron el manejo de la información y la interpretación de los datos, con el propósito de tener resultados claros, de acuerdo a los objetivos propuestos en la investigación.

\section{Resultados y discusión}

Los resultados se exponen en tres apartados, de acuerdo con el proceso de recolección de datos planteados anteriormente. La primera parte, muestra: a) el contexto, b) la percepción de los empresarios en la región sobre el eco marketing, c) su aplicación en las estrategias para el fomento de las diferentes actividades de turismo de naturaleza, d) las cuatro P del marketing mix y, e) las oportunidades de implementación del eco marketing. La segunda, expone la valoración del eco marketing y del turismo de naturaleza y, describe las potencialidades y progresos de esta última actividad; por último, se explica la importancia de la economía ecológica como fundamento de esta nueva tendencia.

3.1. El Contexto. Los empresarios para promover el turismo de naturaleza están constituidos legalmente; en su mayoría, como Personas Naturales, Entidades Sin Ánimo de Lucro (ESAL), y Personas Jurídicas Sociedad Limitada. Sus empresas se caracterizan por contar con una significativa experiencia en el mercado, fueron creadas hace más de cuatro años; tienen como lugares de operación los distintos municipios de departamento de Caquetá; visualizan el turismo de naturaleza como una fuente para dar a conocer la región del Caquetá. Este aspecto, se considera representativo por las grandes oportunidades para la prestación de los servicios turísticos.

Dado el potencial y contribución para la conservación, restauración y protección del ambiente, el turismo de naturaleza fue destacado por parte de los empresarios, como una coyuntura para cambiar las actividades económicas poco amigables con el ambiente. En este sentido, las alternativas analizadas para la promoción del turismo de naturaleza y sus productos complementarios son: a) turismo de aventura, b) ecoturismo, c) agroturismo, d) turismo gastronómico, e) turismo cultural, f) turismo bienestar y, g) turismo científico).

Asimismo, los encuestados percibieron el ecoturismo como la forma de turismo de naturaleza más ofertada en sus empresas, lo promueven con el fin de crear una conciencia ambiental que contribuya al reto del milenio, en aras de propender hacia la minimización de impactos negativos en la naturaleza y la cultura. En este sentido, hay consciencia acerca de la dinámica implicada en la promoción del turismo de naturaleza y la demanda de mayores esfuerzos. Esto se corrobora por el impacto percibido por las comunidades locales ante la oferta del turismo convencional, el cual conlleva la excesiva capacidad de carga y la afectación de lo sociocultural. Para los participantes en el estudio, estas situaciones fueron consideradas como las causantes del detrimento ambiental, al practicarse este tipo de turismo de manera irresponsable, sin prever consecuencias. Asimismo, lo perciben como uno de los más practicados en Colombia, con un impacto muy negativo.

3.2. El Eco marketing. Se indagó sobre el término eco marketing, a través de la presentación de conceptos que definen su contextualización, propósito y enfoque. Los análisis de este interrogante arrojaron el desconocimiento existente por parte de 
los empresarios, quienes no comprenden el significado del término, ni identifican su objetivo principal. En la Figura 1 se presentan estos resultados:

\section{Figura 1. Definición de eco marketing. Fuente: elaboración propia (2019)}

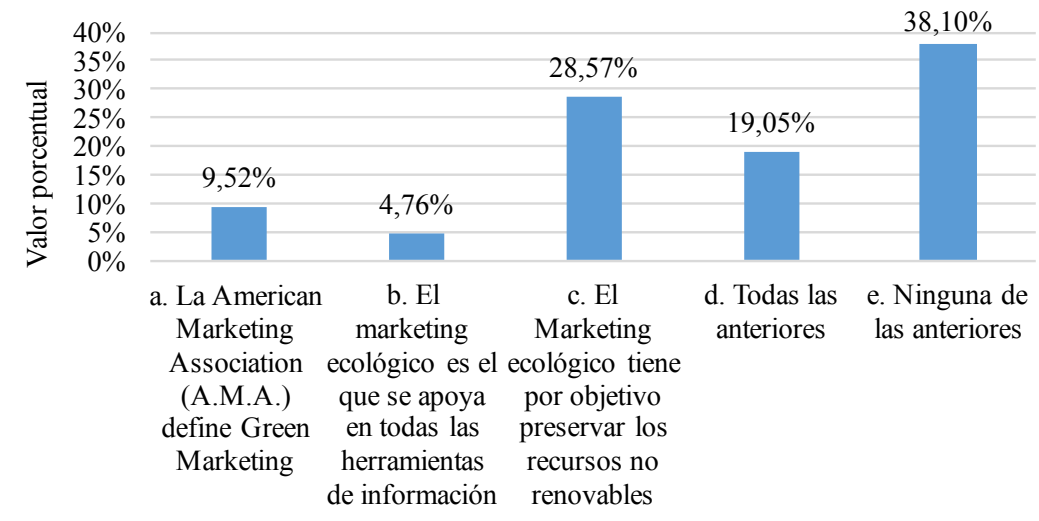

Definiciones de Eco Marketing

Sin embargo, los empresarios de la región, aunque no conozcan el concepto de forma precisa, perciben el marketing como factor trascendental; identifican sus características generales y demás aspectos importantes para prestar adecuadamente los servicios en las empresas. Para superar las falencias de conocimiento que se detectaron, plantean el requerimiento de la realización de talleres, en donde se efectúe el análisis concienzudo del concepto de marketing. Este es entendido como: "un sistema total de actividades de negocios ideado para planear productos satisfactores de necesidades, asignarles precios, promover y distribuirlos a los mercados meta, a fin de lograr los objetivos de la organización" (Stanton, Etzel, y Walker, 2004, p. 7).

En relación con el grado de conocimiento sobre las cuatro 4P's del eco marketing, los empresarios no tienen claridad en el concepto de este término; por tanto, no están en condiciones para generar dinámicas relacionadas con la integración de los elementos de marketing con los retos ambientales. Por otra parte, el 57,1\% implementa estrategias de eco marketing en los procesos organizacionales de las empresas turísticas; el 42,9\% no realiza ninguna acción relacionada con la puesta en marcha de tácticas para contribuir a este tipo de marketing, tal como ilustra en la Figura 2, donde se muestran cada una de las acciones. 
Figura 2. Estrategias de eco marketing en las empresas. Fuente: elaboración propia (2019)

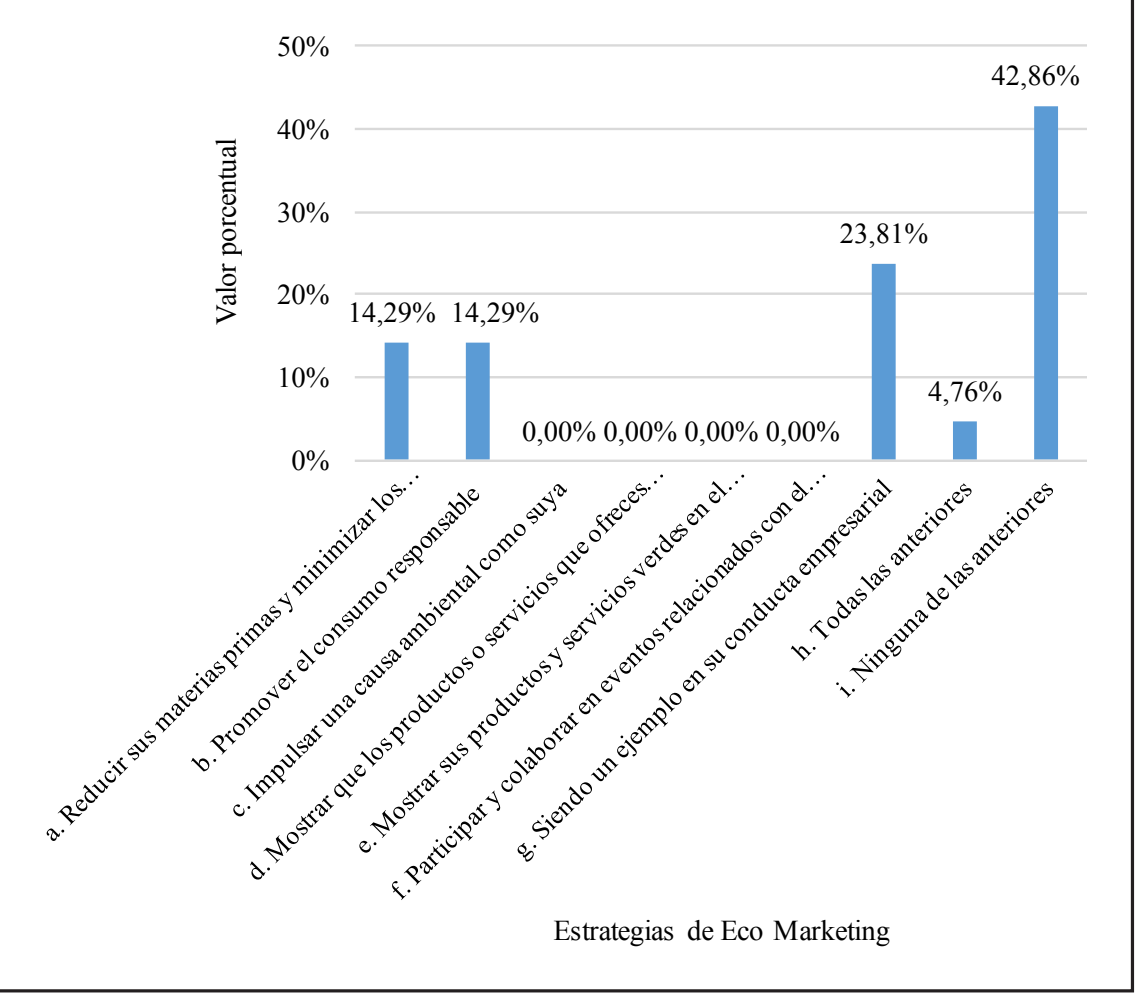

En efecto, las estrategias más destacadas son la reducción de materias para minimizar los residuos generados $(14,29 \%)$ y el impulso del consumo responsable frente al consumismo convencional (14,29\%); dichas acciones son consideradas fundamentales para la vivencia de una conducta empresarial ejemplar en materia ambiental.

Igualmente, los empresarios participantes en la investigación, consideraron que, el poco empleo del eco marketing en el turismo ofertado por sus empresas se debe a la carencia de formación en los diferentes componentes de este tipo de marketing y, por tanto, prestan sus servicios de manera empírica y convencional. Además, otras posibles causas generadoras de una baja implementación del eco marketing en el sector turístico, son: a) la falta de interés, b) el desconocimiento sobre el tema y, c) las debilidades en la planeación financiera. En este tópico, resaltaron la importancia de acceder a una adecuada formación para responder a los retos de los cambios a implementar en pro de la conservación del ambiente.

No obstante el desconocimiento sobre el tema del eco marketing, el 94,44\% de los empresarios están dispuestos a invertir en marketing; son aquellas que creen que la empresa puede crecer con esta estrategia, en aras de facilitar la ejecución de actividades de formación vinculadas con la premisa de avanzar de forma positiva al aplicarlo y así, contribuir al logro del desarrollo sostenible (Estrada-Cely, SánchezCastillo, y Gómez-Cano, 2019); por lo tanto, la relación es directamente proporcional entre el crecimiento de la empresa y la aceptación de las ventajas del marketing; dado que, quien no cree en su aporte en pro del crecimiento de la empresa no invertiría en él, como se indica en la Figura 3: 
Figura 3. Relación crecimiento empresarial y estrategias de eco marketing Fuente: Software SPSS (2019)

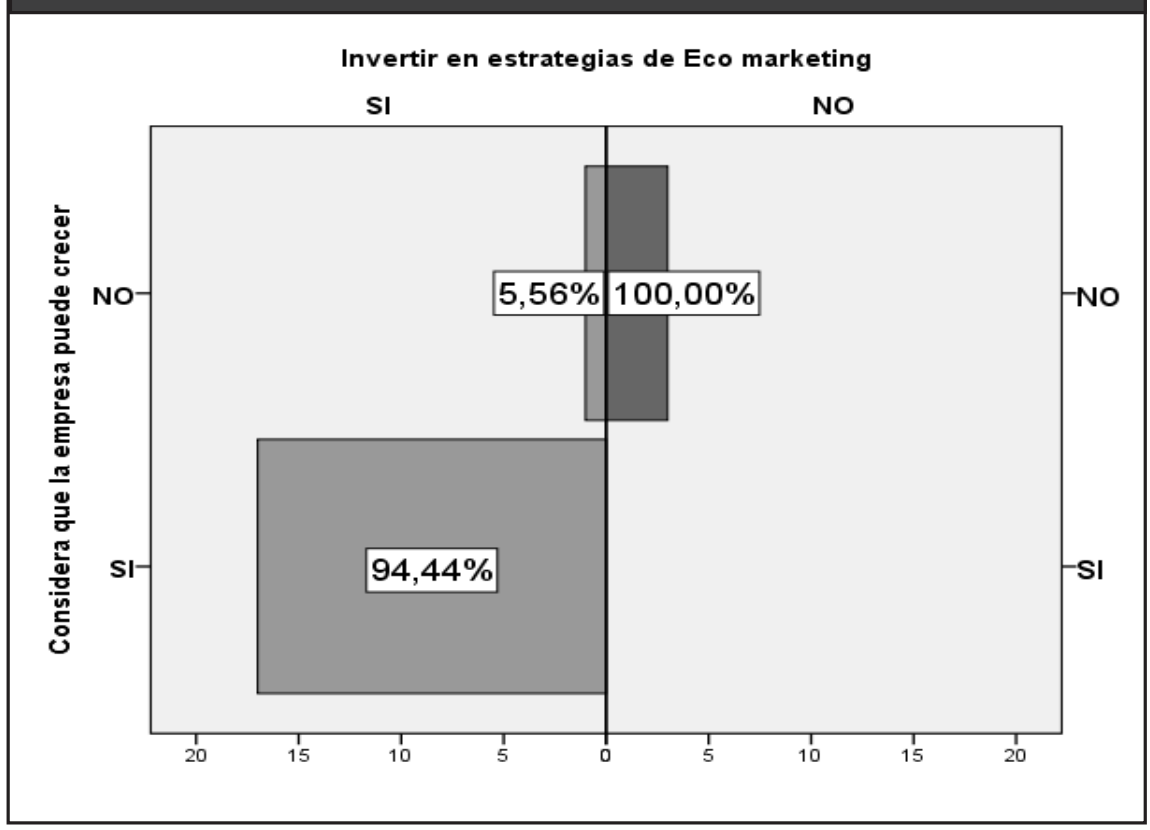

En lo concerniente a las cuatro P del marketing mix, el 47,6\% de los empresarios trabaja en la práctica de todos los pilares claves para lograr los objetivos propuestos; mientras que, el $52,4 \%$ señaló que, sus estrategias están direccionadas hacia los aspectos relacionados con el producto y la promoción; es decir, se enfocan en mostrar los beneficios de los servicios y en buscar los medios para darlos a conocer a nivel local, nacional e internacional. En el caso de las estrategias de marketing para estas cuatro variables, los empresarios destacaron la diversificación e innovación de los servicios turísticos, centradas en ofrecer un portafolio para atraer a muchos consumidores; de igual forma, han mencionado la inclusión de estrategias en función de la estacionalidad (paquetes turísticos especiales) y del segmento de mercado (familias, jóvenes, grupos empresariales, entre otros), con el objetivo de fijar los precios más adecuados. De otro lado, los encuestados señalan la intermediación de diversos agentes para la venta de los servicios. En cuanto a la promoción de estos servicios está enfocada en el empleo de herramientas de marketing modernas (páginas web propias, presencia en redes sociales, anuncios publicitarios en páginas web reconocidas del departamento) y la adopción de modelos empresariales basados en TIC para generar una mayor aproximación hacia los clientes (López, y López, 2018) (Sarmiento, y Ferrão, 2019).

Otro aspecto, de acuerdo con los resultados, es el del mercado objetivo de las empresas, el cual está constituido por los turistas con edades entre 21 y 42 años; estos representan un alto porcentaje de aquellos que acuden para practicar actividades de turismo de naturaleza. Un factor importante a tener en cuenta es el origen de los turistas; al respecto, el 50\% corresponde a aquellos residentes en el departamento y, el restante, corresponde a los habitantes de las principales ciudades de Colombia (Medellín, Bogotá, Cali).

Como beneficios en la implementación del eco marketing, se identificaron: a) la conservación del ambiente, b) la conquista de nuevos mercados, c) la búsqueda de 
una mayor demanda, d) el posicionamiento de la empresa y, e) la perdurabilidad en el entorno de negocios; estos se señalan en la Figura 4:

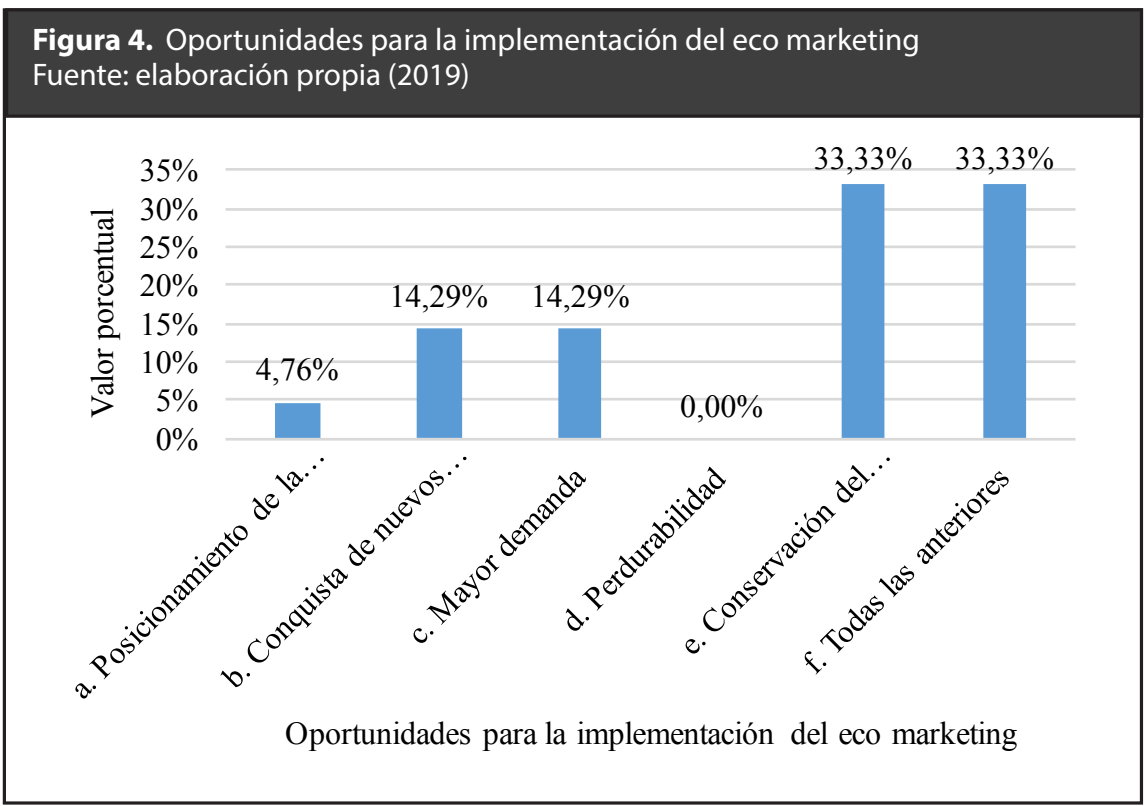

3.2.1. Valoración del eco marketing y del turismo de naturaleza. Los empresarios participantes en el grupo focal consideraron que, por residir en un territorio rico en biodiversidad, se debe apreciar e impulsar el desarrollo del turismo de naturaleza en la región. Además, enfatizaron sobre la importancia de plantear nuevas opciones de inversión y apoyo a los servicios ofrecidos en el Departamento de Caquetá, fundamentados en la existencia de diversos lugares estratégicos para promover el turismo de naturaleza y practicar actividades turísticas (aviturismo, pesca deportiva, senderismo, ecoturismo, turismo rural, turismo de aventura, entre otras). Cabe destacar la percepción clara sobre el concepto de turismo de naturaleza de los empresarios y su reconocimiento como una nueva opción para realizar actividades no convencionales, que permitan, entre otras: a) la interacción de la sociedad, b) la generación de ingresos económicos, c) el mejoramiento de la calidad de vida y, d) un bajo impacto ambiental.

Es así, como los empresarios y directivos, participantes en esta investigación, determinaron la relevancia del eco marketing en los procesos implementados en la oferta de servicios turísticos, los cuales estarán enmarcados en la nueva concepción de cada una de las variables de la mezcla de marketing verde (producto, precio, promoción y plaza). Adicional a esto, señalaron la trascendencia de este tipo de marketing para la sociedad, gracias a las políticas y programas establecidos a nivel mundial. La finalidad es crear conciencia y fortalecer el compromiso social de realizar prácticas amigables desde distintos escenarios para la reducción del impacto ambiental. La perspectiva es la generación de condiciones óptimas para el mercado del turismo de naturaleza en el Departamento de Caquetá.

3.2.2. Potencialidades y progresos del turismo de naturaleza. Según los entrevistados, responsables de ejecutar políticas públicas, se ha avanzado en el desarrollo económico del Caquetá por las potencialidades y progresos en materia de turismo de naturaleza. El posconflicto ha permitido el fortalecimiento del sector turismo a nivel departamental y nacional y, ha propiciado una dinámica empresarial productiva 
más competitiva. A su vez, indican que, gracias al posconflicto, Caquetá adquiere una imagen positiva generadora de confianza para producir interés en los turistas. Por lo anterior, destacaron al departamento como una potencia turística reconocida a nivel nacional, producto del trabajo articulado entre diferentes entidades: Gobernación del Caquetá, Cámara de Comercio Florencia, Pasión Caquetá, Corpoamazonia, Alcaldía de Florencia, entre otras.

Para los directivos entrevistados, la mayor potencialidad del sector turístico es su papel integrador porque puede constituirse en el eje dinamizador de los demás sectores de la economía del Departamento de Caquetá. La necesidad radica en la organización de la prestación de los servicios para la ejecución de las actividades en toda la cadena de valor de la economía colombiana (Estado, agencias de viaje, hoteles, terminales aéreos-terrestres, empresas de transporte, restaurantes, centros comerciales y demás proveedores).

Actualmente, el turismo es una industria considerada como parte de la economía colombiana (Estado, empresas de promotoras del turismo, empresas de servicios, comunidad en general), por el volumen de producción de los bienes y servicios requeridos para la prestación de las actividades. Esto se corrobora con los datos de ingreso de extranjeros; por ejemplo, en el 2015, entraron al país 1.598 .722 extranjeros con motivo de turismo. Esta cifra aumentó a 1.871 .365 en 2016; a 2.521.900, en 2017; y a 3.270.356, en 2018 (Presidencia de la República de Colombia, 2018).

Dada la posibilidad de crecimiento de las empresas mediante la implementación de prácticas de mercadeo con el fin de innovar en la prestación de sus servicios, y obtener ventajas frente a aquellas organizaciones que se dedican al turismo convencional; en el desarrollo de la investigación, se evidenció la necesidad de poner en práctica el eco marketing y sus características para lograr el fomento de las diferentes actividades de turismo de naturaleza. Además, se resaltó la importancia de la promoción y divulgación del turismo de naturaleza, complementado con el eco marketing estratégico. Para tal efecto, se requiere su incorporación en la misión y valores de las empresas constituidas. La implementación de este aspecto, posibilita el tener ventajas competitivas a través de la identificación de estrategias de segmentación y de posicionamiento, con el diseño de planes de marketing verde y, la ejecución de estos planes con control.

Sin embargo, el turismo de naturaleza, aunque ha logrado avances, aún no se posiciona en la economía de la región, prima el turismo convencional. La prospectiva es el aprovechamiento de la dinámica suscitada por la economía cultural y de entretenimiento para que esta situación puede cambiar. En la última década se experimenta en la promoción de un turismo sustentable a partir de impulsar la responsabilidad social con la naturaleza y los valores de la cultura local. En el Departamento del Caquetá se desarrolla el ecoturismo y agroturismo con el compromiso social de las empresas conformadas para ofertar un turismo sustentable y evitar así, impactos ambientales negativos relacionados con el crecimiento de las ciudades y mal uso del suelo, la presión sobre recursos escasos, destrucción de ecosistemas; la contaminación, el deterioro del paisaje y del entorno urbano (Wallace y Russell, 2004).

No obstante, las ventajas enunciadas, no se pueden descartar, los impactos sociales negativos ocasionados por los turistas, al relacionarse con los residentes locales. En este sentido, el Banco Interamericano de Desarrollo (BID) resalta crecimiento de la criminalidad, prostitución y turismo sexual organizado; además de crear cambios en la estructura social tradicional y, la identidad cultural de las comunidades.

La alternativa del eco marketing es vista por parte de los empresarios como potencialidad en la fase exploratoria, según el ciclo de vida del destino turístico, por 
su importancia y necesidad para atraer turistas internacionales y del interior del país que contribuyan al empoderamiento del turismo de naturaleza para la obtención de una mejor calidad de vida de las comunidades. Esta perspectiva, concuerda en varios aspectos de la política pública de Naciones Unidas en relación con el desarrollo sostenible y sus 17 objetivos (Chávarro, et al., 2017); también, con los ámbitos claves, determinados como elementos esenciales para la contribución del turismo de naturaleza: a) la inclusión social, b) empleo y reducción de la pobreza, c) protección ambiental y cambio climático, d) los valores culturales, diversidad y patrimonio y, e) la compresión mutua, paz y seguridad (Organización Mundial del Turismo, 2017). Adicionalmente, se apoya y aboga por el cumplimiento de lo estipulado en esta materia en el Plan Nacional de Desarrollo Pacto por Colombia, pacto por la equidad y el Plan Sectorial de Turismo 2018 - 2022.

Un aspecto importante del eco marketing, consiste en no colocar el rotulo de eco y verde, para aportar al antropocentrismo; por el contrario, su trascendencia radica en la responsabilidad social, integral asociada al turismo, Méndez, y García (2017), que permita la sustentabilidad; una sustentabilidad súper fuerte. Para Gudynas (2010), se caracteriza por un cambio absoluto en la matriz de pensamiento, hacia el biocentrismo. En este entendimiento, la valoración económica de los elementos de la naturaleza convive como una más a ponderar, junto con otras, e incluso, puede ser dejada de lado por valores más importantes; como, por ejemplo, la conservación del patrimonio cultural de un grupo o región: esintra natura. Se trata de la integración de una pluralidad de lógicas y saberes donde la económica convive con otras, más solidarias y equitativas. En esta tesis la visión que predomina es ecosistémica, considerando a las sociedades humanas como un elemento más de interacción constante junto con los otros, tendiente a la subjetivación de la naturaleza.

La sustentabilidad fuerte, deviene de la economía ecológica, que se caracteriza por aspectos insustituibles del patrimonio natural, como el capital natural crítico, que da soporte a la vida y precisa protección. Por su parte, la sustentabilidad débil, se basa en la economía neoclásica y en la sustitución de capital natural (Martínez, y Roca, 2013). La economía ecológica precisa que la tasa de recolección de recursos renovables no exceda su tasa de regeneración y que la tasa de generación de residuos no exceda la capacidad de asimilación (Ramírez, y Antero, 2014). Por ende, la sustentabilidad, respeta la capacidad de renovación de los recursos y la de asimilación de residuos, mantiene constante el acervo de capital natural.

3.3. La Economía Ecológica-SF. Busca la interacción entre las dimensiones económica, social y ambiental. Sus principales herramientas y estrategias son análisis multicriterio y soluciones basadas en la naturaleza; el crecimiento que promueve es cualitativo, donde los conflictos ecológicos son distributivos, con políticas públicas ambientales dirigidas a la conservación e inclusión del componente humano. La economía ecológica es la teoría económico-ambiental que ha logrado incorporar el estudio del aspecto social de las problemáticas ambientales, generadas en torno a la exclusión del factor humano. Es una crítica a la economía neoclásica; la cual parte de la perspectiva de ver a la economía inserta en un sistema mayor, el ecosistema tierra, con recursos finitos, e interrelaciones con el aprovechamiento de recursos naturales y los sistemas sociales y económicos (Martínez, y Roca, 2013).

El eco marketing, a su vez, se fundamenta en la economía ecológica que pretende transformar la política pública ambiental; esta, en su postura neoclásica, ha saqueado los insumos de los ecosistemas. La propuesta es una política que proteja la biodiversidad y se preocupe por la sustentabilidad. Así, las políticas públicas ambientales desde la economía ecológica, son las que, promueven la conservación de los ecosistemas y una sustentabilidad fuerte, no excluyen el elemento social del desarrollo sustenta- 
ble; toda vez que, contemplan a las comunidades y sus beneficios. Por lo tanto, dicha teoría, es idónea para abordar la dimensión humana de problemáticas ambientales. La Sustentabilidad fuerte, se basa en la teoría economía ecológica y la SF es capital natural crítico insustituible.

También, aborda la interrelación entre el aprovechamiento del entorno, el factor humano y la dimensión económica; aspectos que la hacen transdisciplinaria. Significa que es holística, al contemplar dichas propiedades; por lo tanto, los problemas ambientales y las políticas públicas respectivas, no se apartan de los conflictos sociales (Martínez, y Roca, 2013). La economía ecológica al buscar la sustentabilidad, precisa de políticas públicas que, aborden la perspectiva social de los problemas ambientales, como los conflictos ecológicos; esto conlleva a incluir el estudio de los movimientos sociales ecologistas.

En síntesis, la economía ecológica se sustenta en las interrelaciones entre los sistemas económico, ambiental y social, a fin de lograr un equilibrio entre dichos sistemas y abordar el carácter social de los problemas ambientales. En esta óptica, postula a diferencia de las otras teorías, una vida dentro de los límites ambientales del planeta, para contrarrestar la desigualdad en la apropiación de recursos. La economía ecológica reconoce la existencia de recursos naturales insustituibles y esenciales para la supervivencia humana, como los eco-sistemas (capital natural crítico) y se vincula con las soluciones basadas en la naturaleza; implementadas también, por la economía verde.

Con estos basamentos, un factor considerado fundamental y relevante para potenciar el desarrollo del turismo de naturaleza es la formación en la temática del eco marketing. En este sentido, la propuesta es la realización de talleres de manera participativa con los agentes sociales interesados en su promoción. Su implementación se propone a través de la realización de los siguientes talleres: a) fundamentos de marketing, b) introducción, contexto y concepto de eco marketing, c) cuatro 4P's del eco marketing, d) rol del consumidor verde, e) estrategias de posicionamiento, f) estrategias de marketing digital, g) estrategias de redes sociales, h) turismo de naturaleza, i) legislación para ofertar servicios de turismo de naturaleza, j) análisis del mercado, k) impacto del eco marketing y el turismo de naturaleza para el ambiente.

La propuesta de formación se fundamenta en el análisis de las necesidades identificadas en la investigación, con el fin de sensibilizar y orientar a los integrantes de la cadena de valor del turismo de naturaleza. De igual manera, para promover la ejecución de actividades que contribuyan a la dinamización de la economía, el posicionamiento de las empresas, la protección del ambiente, el reconocimiento del Departamento del Caquetá y la mitigación de problemas ambientales detectados en la región, dentro de los que se destaca la deforestación. Asimismo, para el desarrollo de los talleres, se requerirá del trabajo articulado con la institucionalidad de la región (en este caso: Ministerio de Comercio, Industria y Turismo, Cámara de Comercio de Florencia, Servicio Nacional de Aprendizaje - SENA, Corporación para el Desarrollo Sostenible del Sur de la Amazonia - Corpoamazonia, Gobernación del Departamento de Caquetá, Alcaldía del Municipio de Florencia, Universidades).

Si bien es cierto hay trabajos de investigación relacionados como el de Córdova (2012) que, coincide con la importancia de la motivación de los diversos actores para la implementación del eco marketing en las estrategias para el turismo; el de Martínez (2017) quien propone la aplicación de técnicas avanzadas del marketing turístico digital y, en esta perspectiva, el de Fandiño, Dávila, y Rodríguez (2018) quienes encontraron como requerimientos de las empresas de turismo de naturaleza la formación para ampliar los conocimientos sobre los beneficios y ventajas del uso de las TIC y redes sociales; es importante, la identificación de futuras temáticas 
de investigación, es el principal reto para la profundización y generación de nuevos conocimientos e involucrar a un mayor segmento de la sociedad.

Finalmente, el aporte de esta investigación constituye un elemento esencial para potencializar el turismo de naturaleza a través de la aplicabilidad del eco marketing y, se convierte en la oportunidad precisa para que los empresarios mejoren la prestación de los servicios turísticos y los directivos participen en la creación de políticas públicas para fortalecer el novedoso turismo amigable con el ambiente y dinamizar la economía de la región.

\section{Conclusiones}

- El eco marketing es una alternativa para las empresas, con el fin de satisfacer las necesidades de los consumidores y crear una tendencia enfocada al respeto por el ambiente y el gusto por los productos ecológicos, naturales y culturales; su materialización representa la adaptación continua a los nuevos retos del entorno cambiante.

- Los empresarios identifican el eco marketing como una tendencia que trasciende a un escenario fundamental para la sociedad por la potencialización del turismo de naturaleza mediante su implementación. Este se puede convertir en el eje transversal para conseguir un resultado impactante a nivel departamental, nacional e internacional. Desde el enfoque del respeto hacia la naturaleza y conservación del ambiente, se aspira aportar a la consecución del mejoramiento en las condiciones económicas, ecológicas y culturales.

- La aplicación de estrategias basadas en el eco marketing requiere del apoyo de los empresarios, en aras de buscar el mejoramiento de los procesos de producción de servicios turísticos y la generación de cambios de actitud que permitan minimizar los efectos adversos para la comunidad.

- Igualmente, recobra mucha importancia la disposición para implementar en las organizaciones (fundaciones, empresas, asociaciones comunitarias) la promoción turística de actividades y acciones pertinentes mediante el eco marketing; esto marca el punto de partida para clasificarlas como ejecutoras de estrategias que posibiliten la responsabilidad social y la conservación del ambiente.

- El objetivo principal de las empresas del sector turístico será convertir a las regiones, en particular al Caquetá, en un destino favorable para los viajeros nacionales e internacionales mediante la oferta de alternativas de turismo de naturaleza a través del enfoque del eco marketing. La perspectiva es el mejoramiento de la calidad de vida y de las condiciones socioeconómicas de sus habitantes, a través de la protección y cuidado con responsabilidad social de los escenarios naturales del territorio.

- Los aportes del eco marketing y la economía ecológica serán tendencia a nivel mundial para favorecer la implementación de estrategias en procura de satisfacer las necesidades de los consumidores y velar por la protección del ambiente. Esta tendencia se puede catalogar como un nuevo paradigma en el mundo empresarial con grandes desafíos para la sociedad. 
- La formación en temáticas relacionadas con el eco marketing es una condición clave para promoverlo; se constituye en una necesidad apremiante que requiere el apoyo y la articulación de la institucionalidad.

Como conclusión global de la investigación y, según el objetivo expuesto en este artículo, se determina que, existe un contexto favorable y un alto grado de interés de los empresarios para propiciar las condiciones que conlleven al impulso el eco marketing enfocado hacia la oferta de productos y servicios de turismo de naturaleza en el departamento de Caquetá.

\section{Referencias}

Aguilar, S.; Barroso, J. (2015). La triangulación de datos como estrategia en investigación educativa. Revista de Medios y Educación, 47, julio, pp. 73-88.

American Marketing Association, Committee on Definitions (1960). Marketing Definitions: A Glossary Of Marketing Terms, Estados Unidos, Editorial Chicago.

Baker, W.E.; Sinkula, J.M. (2005). Environmental marketing strategy and firm performance effects on new product performance and market share. Journal of the Academy of Marketing Science, 33(4), pp. 461-475. [Online]. Disponible en: https://doi. org/10.1177/0092070305276119

Bradley, N. (2013). Marketing Research: Tools and Techniques, Estados Unidos, Oxford University Press.

Bueno-Solano, V.M.; Wendlandt-Amézaga, T.R.; Valenzuela-Reynaga, R. (2018). Personalidad de marca en instituciones de educación superior en un municipio del noroeste de México. Clío América, 12(24), julio-diciembre, pp. 184-197. [Online]. Disponible en: https:// doi.org/10.21676/23897848.2864

Calomarde, J. V. (2000). Marketing Ecológico, Madrid, Editorial Pirámide.

Castañeda, T. (2017). La influencia del eco marketing desde la perspectiva de consumo en mercados orgánicos de México, tesis (Pregrado en Mercadotecnia), Guadalajara Jalisco, Universidad de Guadalajara, Centro Universitario de Ciencias Económico Administrativas, 77 pp. Disponible en: https://www.researchgate.net/publication/321493150_La_ Influencia_del_Eco_marketing_desde_la_perspectiva_de_consumo_en_mercados_organicos_de_Mexico

Castellano, S.; Urdaneta, J.A. (2015). Estrategias de mercadeo verde utilizadas por empresas a nivel mundial. Revista de Estudios Interdisciplinarios en Ciencias Sociales, 17(3), septiembre-diciembre, pp. 476-494.

Córdova, V.H. (2012). Marketing ecológico y turismo sostenible en la provincia del Tungurahua - Ecuador, desde una perspectiva estratégica, tesis (Doctorado en Ciencias de la Empresa), España, Universidad Rey Juan Carlos, Facultad de Ciencias Jurídicas y Sociales, 339 pp. Disponible en: https://dialnet.unirioja.es/servlet/tesis?codigo=112589

Cruz, C.; Enciso, G.; Forero, S.; Garzón, C.; Hoyos, R.; Leyva, K.; Linero, J.; López, S.; Maguiña, O.; Neme, S.; Pereira, C.; Riveros, G.; Zitzmann, J. (2017). Fundamentos de Mercadeo, Bogotá, Ecoe Ediciones.

Chamorro, A. (2001). Marketing ecológico; sí, marketing ecológico, Badajoz, Universidad de Extremadura.

Chamorro, A.; Miranda, FJ.; Rubio, S. (2006). El estado de la investigación sobre marketing ecológico en España: análisis de revistas españolas 1993-2003. Investigaciones Europeas de Dirección y Economía de la Empresa, 12(2), julio, pp. 137-156.

Chávarro, D.; Vélez, M. I.; Tovar, G.; Montenegro, I.; Hernández, A.; Olaya, A. (2017). Los Objetivos de Desarrollo Sostenible en Colombia y el aporte de la ciencia, la tecnología y la 
innovación, Colombia. Departamento Administrativo de Ciencia, Tecnología e Innovación (Colciencias). Disponible en: https://www.colciencias.gov.co/sites/default/files/ objetivos_de_desarrollo_sostenible_y_aporte_a_la_cti_v_3.5.pdf

De Ruyter, K.; De Jong, A.; Wetzels, M. (2009). Antecedents and consequences of environmental stewardship in boundary- spanning B2B teams. Journal of the Academy of Marketing Science, 37(4), pp. 470-487. [Online]. Disponible en: https://doi.org/10.1007/ s11747-009-0138-0

Díaz-Sarmiento, C.; López-Lambraño, M.; Roncallo-Lafont, L. (2017). Entendiendo las generaciones: una revisión del concepto, clasificación y características distintivas de los baby boomers, X y millennials. Clío América, 11(2), julio-diciembre, pp. 188-204. [Online]. Disponible en: https://doi.org/10.21676/23897848.2440

Díaz, J.; Moya, O. (2016). Impacto de las actividades de green marketing, la contribución al proceso de creación de valor de marca y el impacto en la intención de compra en el sector tecnológico empresarial en Bogotá, tesis (Pregrado en Mercadeo), Colombia, Universidad Santo Tomás, Facultad de Mercadeo, 136 pp. Disponible en: https:// repository.usta.edu.co/bitstream/handle/11634/2490/D\%C3\%ADazortegajorge. pdf?sequence $=2$

Dima, I.; Vlăduțescu, S. (2012). Persuasive communication in logistic negotiation. International Journal of Economical Research, 3(1), pp. 14-21.

Durmaz, Y.; Sevil, Z. (2011). A theoretical approach to the concept of green marketing. Interdisciplinary Journal of Contemporary Research in Business, 3(2), pp. 1808-1814.

Estrada-Cely, G.E.; Sánchez-Castillo, V.; Gómez-Cano, C.A. (2019). Bioética y desarrollo sostenible: entre el biocentrismo y el antropocentrismo y su sesgo economicista. Clío América, 12(24), julio-diciembre, pp. 255-267. [Online]. Disponible en: http://dx.doi. org/10.21676/23897848.2999

Fandiño, J.R.; Dávila, L.; Rodríguez, M. (2018). Marketing digital en las empresas de Turismo de Naturaleza del Departamento de Magdalena. Vincula Tégica, 4(1), enero, pp. 289296.

Fernández, V. (2015). Marketing mix de servicios de información: valor e importancia de la P de producto. Bibliotecas anales de investigación, 11, pp. 64-78.

Fuller, D.; Butler, D. (2015). Eco-Marketing: A Waste Management Perspective. En: Wilson E., Black W., Proceedings of the 1994 Academy of Marketing Science (AMS) Annual Conference, 1a ed., Cham, Switzerland, Springer International Publishing AG. Disponible en https://doi.org/10.1007/978-3-319-13162-7_89

García, B.; Salvaj, E. (2017). Asociacionismo, redes y marketing en la transformación hacia el turismo experiencial. El caso del barrio de las Letras. Madrid. Cuadernos de Turismo, 40, pp. 315-338. [Online]. Disponible en: http://dx.doi.org/10.6018/turismo.40.309731

Gil, C. (2019). Del turismo inmobiliario al turismo de naturaleza ¿cambio de modelo o cambio De Discurso? El Caso De Noja, Un Municipio En La Costa Cantábrica. Cuadernos De Turismo, 43, pp. 301-324. [Online]. Disponible en: http://dx.doi.org/10.6018/turismo.43.12

Gómez, J.F. (2017). El turismo de naturaleza como oferta turística innovadora en el contexto del desarrollo sostenible en la región San Martín, tesis (Doctorado en Turismo y Hotelería), Perú, Escuela Profesional de Turismo y Hotelería, Facultad de Ciencias de la Comunicación, Turismo y Psicología, 318 pp. Disponible en: http://www.repositorioacademico.usmp.edu.pe/bitstream/handle/usmp/2655/gomez_rj;jsessionid=7EABE4F F649447349D01479D7927CDE3?sequence=1

Grinstein, A.; Nisan, U. (2009). Demarketing, minorities, and national attachment. Journal of Marketing, 73(2), pp. 105-122. [Online]. Disponible en: https://doi.org/10.1509/ jmkg.73.2.105

Gudynas, E. (2010). Desarrollo sostenible: una guía de conceptos y tendencias hacia otra economía. Otra Economía, 4(6), enero-junio, pp. 43-66.

Hair, J.; Bush, R.; Ortinau, D. (2010). Investigación de Mercados, Ciudad de México, McGrawHill. 
Hernández, R.; Fernández, C.; Baptista, P. (2014). Metodología de la Investigación, Ciudad de México, McGraw-Hill.

Hueso, A.; Cascant, J. (2012). Metodología y técnicas cuantitativas de investigación, Valencia, Editorial Universidad Politécnica de Valencia.

Instituto de Estudios Sociales de Andalucía (2012). La triangulación metodológica en el ámbito de la investigación social: dos ejemplos de uso. [Online]. Disponible en: https:// core.ac.uk/download/pdf/36126393.pdf

Instituto de Hidrología, Meteorología y Estudios Ambientales - IDEAM (2019). Boletín de Detecciones Tempranas de Deforestación (17). [Online]. Disponible en: http://www. ideam.gov.co/documents/24277/84382637/Detecciones+Tempranas+de+Deforestaci \%C3\%B3n/96e81976-195e-4d0f-8aaf-24c05c7312f8

Kotler, P.; Keller, K. (2006). Dirección de Marketing, Ciudad de México, Pearson Educación.

Kotler, P.; Armstrong, G. (2012). Marketing, Ciudad de México, Pearson Educación.

Kotler, P.; Armstrong, G. (2017). Fundamentos de Marketing, Ciudad de México, Pearson Educación.

López, A.L.; López, S.A. (2018). Impacto de las TIC en el turismo: caso colombiano. Cuadernos de Turismo, 41, enero-junio, pp. 399-418. [Online]. Disponible en: http://dx.doi. org/10.6018/turismo.41.327081

López, D.D.; Moya, F. (2015). Marketing ecológico como estrategia para mejorar la percepción sobre la minería del carbón en la región Zuliana. Revista Interdisciplinar de Estudios en Ciencias Básicas e Ingenierías, 2(1), enero-junio, pp. 43-53.

López-Vargas, J.C.; Cárdenas-Aguirre, D.M. (2018). Factores de influencia en la coordinación logística para la preparación y atención de desastres - Una revisión de literatura. Revista EIA, 15(30), julio-diciembre, pp. 41-56. [Online]. Disponible en: https://doi.org/10.24050/reia.v15i30.1146

Lorenzo, M.M. (2002). Marketing ecológico y sistemas de gestión ambiental: conceptos y estrategias empresariales. Revista Galega de Economía, 11(2), julio-diciembre, pp. 1-25.

Macris, M. (2013). Labor international circulation within the present day context of globalized economy. International Journal of Management Sciences and Business Research, 2(3), marzo, pp. 33-48.

Machín, M. (2007). Gestión empresarial. Desafíos y oportunidades desde la perspectiva de la gestión ambiental. Revista Futuros, 5(17), pp. 1-10.

Malhotra, N. (2016). Investigación de Mercados, Ciudad de México, Pearson Educación.

Martínez, J.; Roca, J. (2013). Economía ecológica y política ambiental, Ciudad de México, Fondo de Cultura Económica.

Martínez, V. (2017). El turismo de naturaleza: un producto turístico sostenible. Arbor, 193, (785), julio-septiembre, p. a396. [Online]. Disponible en: http://dx.doi. org/10.3989/arbor.2017.785n3002

Méndez, J.C.; García, J.L. (2017). Hacia la responsabilidad integrada del turismo a través de la responsabilidad social empresarial. Estudios Turísticos, 211-212, enero-junio, pp. 57-69.

Monteiro, T.A.; Giuliani, A.C.; Cavazos-Arroyo, J.; Pizzinatto, N.K. (2015). Mezcla del marketing verde: una perspectiva teórica. Cuadernos del CIMBAGE, 17, pp. 103126.

Niño, V. (2011): Metodología de la investigación, Bogotá, Ediciones de la U. 
Ñaupas, H.; Mejía, E.; Novoa, E.; Villagómez, A. (2014). Metodología de la investigación. Cuantitativa - cualitativa y redacción de tesis, Bogotá, Ediciones de la U.

Organización Mundial del Turismo-OMT (2017). Turismo sostenible para el desarrollo. Educación para el desarrollo sostenible. [Online]. Disponible en: http://www. unesco.org/new/es/education/themes/leading-the-international-agenda/education-for-sustainable-development/education-for-sustainable-development/

Otzen, T.; Manterola, C. (2017). Técnicas de muestreo sobre una población a estudio. International Journal of Morphology, 35(1), pp. 227-232. [Online]. Disponible en: http://dx.doi.org/10.4067/S0717-95022017000100037

Patton, M. (2002). Qualitative research and evaluation methods, Estados Unidos, SAGE Publications.

Peña, D.D.; Serra, A.; Cardona, J.R. (2017). Perfil característico y responsabilidad social empresarial del sector hotelero de la región Caribe colombiana. Pensamiento y Gestión, 43, julio-diciembre, pp. 128-149. [Online]. Disponible en: http:// dx.doi.org/10.14482/pege.43.10585

Presidencia de la República de Colombia (2016). Acuerdo final para la terminación del conflicto y la construcción de una paz estable y duradera. [Online]. Disponible en: http://www.altocomisionadoparalapaz.gov.co/procesos-y-conversaciones/Documentos\%20compartidos/24-11-2016NuevoAcuerdoFinal.pdf

Presidencia de la República de Colombia (2018). Plan Nacional de Desarrollo 20182022: Pacto por Colombia, pacto por la equidad. [Online]. Disponible en: https:// www.dnp.gov.co/Plan-Nacional-de-Desarrollo/Paginas/Bases-del-Plan-Nacional-de-Desarrollo-2018-2022.aspx

Ramírez, V.; Antero, J. (2014). Evolución de las teorías de explotación de recursos naturales: hacia la creación de una nueva ética mundial. Luna Azul, 39, juliodiciembre, pp. 291-313.

Rubio-Guerrero, G. (2015). La responsabilidad social empresarial en las instituciones financieras de Ibagué: un análisis multivariante. Revista EIA, 13(25), enero-junio, pp. 119-134. [Online]. Disponible en: http:/dx.doi.org/10.14508/ reia.2016.13.25.119-134

Sánchez, J.A.; Montoya, L.A. (2016). Factores que afectan la confianza de los consumidores por las compras a través de medios electrónicos. Pensamiento y Gestión, 40, pp. 159-183. [Online]. Disponible en: http://dx.doi.org/10.14482/ pege. 40.8809

Sarmiento, J.R.; Ferrão, A.J. (2019). La e-experiencia de marca a través de los medios sociales y su influencia en la e-calidad de la relación y la e-fidelización. Análisis empírico en los sitios web de viajes. Cuadernos de Turismo, 44, julliodiciembre, pp. 351-380. [Online]. Disponible en: http://dx.doi.org/10.6018/ turismo.44.404891

Serrano-Abreo, E.R.; Castellanos-Granados, H.C. (2019). Estudio comparativo de herramientas software libre para la Gestión de Procesos de Negocio. Revista EIA, 16(31), enero-junio, pp. 171-187. [Online]. Disponible en: https://doi. org/10.24050/reia.v16i31.1148

Stanton, W.; Etzel, M.; Walker, B. (2004). Fundamentos de Marketing, Ciudad de México, McGraw-Hill, p. 7.

Talavera, F. J. (2017). Evaluación del impacto del marketing mix en las ventas y rentabilidad de una tienda de artículos deportivos en el Centro Comercial Plaza Norte en el periodo 2015 - 2016, tesis (Maestría en Administración de Negocios), Perú, Universidad Ricardo Palma, Escuela de Posgrado, 103 pp. Disponible 
en: http://repositorio.urp.edu.pe/bitstream/handle/URP/1438/FJTALAVERAC. pdf?sequence $=1 \&$ isAllowed $=y$

Trespalacios, J.A.; Vázquez, R.; De La Ballina, F. J.; Suárez, A. (2016). Investigación de mercados, Madrid, Ediciones Paraninfo.

Vicente, M.A.; Ruiz, M. (2002). Factores determinantes de la integración de la variable medio ambiente en los planteamientos de la economía de la empresa y el marketing. Cuadernos de Gestión, 1(2), enero, pp. 71-84.

Vlăduțescu, S. (2012). The Emphasis of Negative Journalism in the Economic Communication, one of the Consequences of the Global Economic Crisis. Romanian Statistical Review Supplement, 60(4), noviembre, pp. 121-126.

Wallace, G.; Russell, A. (2004). Eco-cultural tourism as a means for the sustainable development of culturally marginal and environmentally sensitive regions. Tourist Studies, 4(3), pp. 235-254. [Online]. Disponible en: https://doi. org/10.1177\%2F1468797604057326 